\title{
Can light make us bright? Effects of light on cognition sleep
}

\author{
Sarah Laxhmi Chellappa ${ }^{\dagger \star \star}$, Marijke C. M. Gordijn ${ }^{\S *}$ and Christian Cajochen ${ }^{\dagger}$ \\ $\dagger$ Centre for Chronobiology, Psychiatric Hospital of the University of Basel, Basel, Switzerland \\ \$ The CAPES Foundation/Ministry of Education of Brazil, Brasilia-DF, Brazil \\ $\S$ Department of Chronobiology, Centre for Life Sciences, University of Groningen, Groningen, The Netherlands
}

\begin{abstract}
Light elicits robust nonvisual effects on numerous physiological and behavioral variables, such as the human sleep-wake cycle and cognitive performance. Light effects crucially rely on properties such as dose, duration, timing, and wavelength. Recently, the use of methods such as fMRI to assess light effects on nonvisual brain responses has revealed how light can optimize brain function during specific cognitive tasks, especially in tasks of sustained attention. In this chapter, we address two main issues: how light impinges on cognition via consolidation of human sleep-wake cycles; and how light directly impacts on sleep and cognition, in particular, in tasks of sustained attention. A thorough understanding of how light affects sleep and cognitive performance may help to improve light settings at home and at the workplace in order to improve well-being.
\end{abstract}

Keywords: light; cognition; sleep; circadian clock; human.

\section{Introduction}

The 24-h reoccurrence of light and darkness represents the most systematic time cue on earth. Thus, it is not surprising that all living organisms integrated the light-dark cycle in their physiology and optimally adapted their anatomy and behavior to anticipate dawn and dusk. In humans, light is intuitively linked with an alert or wakeful state, whereas in nocturnal animals, the light phase

*Corresponding author. comprises the rest phase, thus representing a different temporal niche for sleep. The human visual system is designed to accommodate the needs of a diurnal species from a visual perspective. However, also the nonvisual responses to light point to the role of light as the mediator of inducing daytime physiology in humans. Hormonal secretion, heart rate, body temperature, sleep propensity, alertness, pupillary constriction, and gene expression are all immediately influenced by light-or even hours after light exposure ended-in order to pursue optimal adaptation to 
the imposed light-dark cycle (Berson, 2003; Bromundt et al., 2010; Cajochen et al., 2005; Hatori and Panda, 2010; Lavoie et al., 2003; Muñoz et al., 2005).

Recently, these long-term and acute effects of light on physiology have been described as belonging to the non-image forming (NIF) system, given that these responses are not associated with the classical involvement of rod and cone photopigments (Guler et al., 2008). This has opened a new area of research, which, apart from the role of light in regulating circadian rhythms, focuses on the physiological and anatomical underpinnings of light in modulating sleep and cognition.

Thus, in this chapter, we address two issues: (1) how light impinges on cognition via consolidation of human sleep-wake cycles and (2) how light directly impacts on sleep and cognition, in particular, in tasks of sustained attention.

\section{Effects of light on the circadian timing system and sleep-wake cycles}

The response of the central circadian pacemaker located in the suprachiasmatic nuclei $(\mathrm{SCN})$ to light pulses plays a crucial role in the synchronization to the environmental light-dark cycles. Light pulses presented during the subjective day rapidly induce expression of the immediate early gene c-fos (Rusak et al., 1990) and the clock gene Per1 within the SCN (Albrecht et al., 2001; Edelstein et al., 2003), resulting in phase shifts of behavioral circadian rhythms. Thus, the mamcomponents of the circadian oscillator but also are involved in resetting of the circadian clock (for a review, see Oster et al., 2002). In humans, exposure to light late in the biological day (dusk) leads to a delay in human sleep onset, while exposure to light early in the biological day (dawn) advances activity onset (for a review, see Czeisler and Gooley, 2007). This phase-shifting property of light denotes a clear NIF effect of light, which relies on circadian phase and substantially impacts on the temporal organization of sleep and wakefulness.

Thus, light as the major "Zeitgeber" (i.e., time giver) is a prerequisite for ideal synchronization between the temporal organization of sleep and wakefulness and the external light-dark cycle. As a consequence, attenuated Zeitgeber strength (i.e., not enough light, or light of an inappropriate wavelength, i.e., light at longer wavelengths, $>600 \mathrm{~nm}$ ) and light at inappropriate times (i.e., during the biological night) can lead to improper entrainment between internal (i.e., circadian) and external (i.e., 24-h earth rotation) time, which often occurs in people working on rotating shifts and/or older individuals as well as visually impaired people. There is ample evidence that timed light exposure is a successful countermeasure of circadian misalignment in shift work (Burgess et al., 2002). Exposure to bright light did not only stabilize sleep-wake rhythms in demented older people, but significantly attenuated the decline in mental capabilities over the investigated time span of 4.5 years (Riemersmavan der Lek et al., 2008). Similarly, bright light promoted circadian alignment and prevented the detrimental effects of night work on sustained attention, as measured in an increased response speed on the psychomotor vigilance test (Santhi et al., 2008). The beneficial effects of light on circadian entrainment clearly carry on to cognitive performance. Thus, in this way, it is rather the stabilization of sleep-wake rhythms that leads to better brain function, than the exposure to bright light per se. Strong evidence for this stems from the fact that, if sleep and wakefulness occur out of phase with internal biological time, this impairs several cognitive functions such as learning in humans (Wright et al., 2006). Further, fragmentation of the rest-activity rhythm correlates with age-related cognitive deficits (Oosterman et al., 2009). Thus, stable and consolidated circadian sleep-wake rhythms are an essential requirement for proper cognitive functioning in health and disease, and light is only a means to an end. However, there is recent evidence that light per se may 
directly impinge on sleep, alertness, cognitive performance, and even mood levels probably even without its action via the central circadian pacemaker.

\section{Light directly impacts on sleep and cognition}

In nocturnal animals, there is recent evidence that the acute light-induction of sleep is mediated by melanopsin-based photoreception (Lupi et al., 2008; Tsai et al., 2009), potentially reflecting an additional clock-independent photic input to sleep. To our knowledge, a similar wakefulnessinducing effect of light during sleep has not been substantiated as such in the "diurnal" humans, as it is difficult to apply light while sleeping, and testing the effects of short-wavelength light during sleep is impossible because of the filtering properties of the eyelids (Moseley et al., 1988). Therefore, most of the evidence of acute light effects on sleep in humans comes from studies applying light shortly before or after sleep. Bright light in the morning has been shown to shorten sleep duration (Dijk et al., 1989) and advance circadian rhythms (i.e., melatonin profile), without effects on nonrapid eye movement (NREM) sleep homeostasis (Carrier and Dumont 1995; Dijk et al., 1989). An artificial dawn in the morning during the last 30 min of sleep caused more superficial sleep along with a faster decline in skin temperatures and less sleepiness after waking up (Werken et al., 2010). Despite positive effects on alertness in the morning, the use of the same artificial dawn during 2 weeks did not induce a significant change in circadian phase (Giménez et al., 2010). Bright light in the evening may lead to an increase in sleep latency to NREM sleep stage 2 (Cajochen et al., 1992; Carrier and Dumont 1995) and changes in the temporal dynamics of electroencephalographic (EEG) slow-wave activity (SWA), such that SWA is lower during the first and higher during the fourth NREM-REM sleep cycle as compared to dim light condition (Cajochen et al., 1992). Exposure to bright polychromatic light (2500 lux) in the morning $(06: 00 \mathrm{~h}-09: 00 \mathrm{~h})$ or in the evening (18:00 $\mathrm{h}-21: 00 \mathrm{~h}$ ) for 3 consecutive days can result in earlier sleep termination following morning light than after evening light (Gordijn et al., 1999). Interestingly, the duration of the first REM sleep episode was longer after morning light than after evening light, most likely due to a phase advance of the circadian influence on REM sleep production. This is supported by the observed advance of the circadian rhythm of melatonin. Thus, both sleep termination and REM sleep duration can be manipulated by light exposure. All these effects have been interpreted as reflecting a carryover effect of light's alerting action into sleep (i.e., longer sleep latencies, reduced SWA in the first cycle with an intrasleep rebound of SWA in the last cycle), reflecting the repercussion of the immediate induction of a circadian phase advance or delay on the following sleep episode, and showing the positive effects of artificial dawn on the dissipation of sleep inertia after awakening, which could not be explained by circadian mechanisms.

\section{Circadian and homeostatic influences on the alert state}

Wakefulness is a construct associated with high levels of environmental awareness, which can be tracked down by a wide array of responses, such as subjective perception, and behavior, subcortical and cortical activity (Buysse et al., 2003). Basically, it comprises self-reported low levels of fatigue or sleepiness, fast and more accurate responses in tasks of sustained attention, low power densities in the EEG theta frequency range $(4-8 \mathrm{~Hz})$, and high power densities in the EEG beta frequency range $(12-30 \mathrm{~Hz}$; Badia et al., 1991; Daurat et al., 2000). Subjective perception of wakefulness crucially relies on a time-of-day dependency, to the extent that diurnal fluctuations of alertness follow similar dynamics of core body temperature (CBT), with 
its maximum in the evening and nadir in the early morning (Kleitman, 1987).

Two important protocols have been developed in order to dissect out the relative contributions of circadian and sleep-wake homeostatic processes in humans: the constant routine and the forced desynchrony protocols (Duffy and Dijk, 2002). In the first, the amplitude and phase of many circadian rhythms can be elucidated without the masking effects of food, posture, light, and so forth. In the second protocol, subjects live on artificially very long or very short days so that the circadian system is no longer entrained to the imposed sleep-wake cycle. The desynchronized/ synchronized subjects sleep at different circadian phases of the entire 24-h cycle, which enables to differentiate the contribution of the sleep homeostatic process or the circadian system to a given variable (Dijk et al., 1997). Usually-but not always - both factors contribute substantially to measures as alertness, mood, and neurobehavioral performance (Cajochen et al., 1999a, b; Koorengevel et al., 2003). Accordingly, some forced desynchrony studies have revealed that the deleterious effects of prior wakefulness on alertness were strongest during the minimum of the endogenous CBT rhythm. This strongly indicates that optimal levels of alertness can be achieved when the phase relationship between the endogenous circadian timing system and the sleep-wake cycle is such that the former opposes the wake-dependent deterioration of alertness and performance, as conceptualized in the "twoprocess" model (Borbély 1982; Daan et al., 1984). The most effective means of obtaining this occurs when the waking day starts $\sim 2 \mathrm{~h}$ after the endogenous circadian minimum of CBT rhythm, which corresponds to $\sim 2 \mathrm{~h}$ after the circadian maximum of the plasma melatonin rhythm. Taken together, this implies that the circadian process plays a wake-promoting role that counteracts the accumulating homeostatic drive for sleep during wakefulness. The result of the interaction between these two fundamental properties emanating from the central nervous system is that humans are able to maintain alert wakefulness for $\sim 15-17 \mathrm{~h}$ (Czeisler et al., 1994; Dijk and Czeisler 1994). Considering the temporal dynamics of these processes on alertness, one can hypothesize that light exerts its alerting effects most strongly when the circadian drive for sleep is at its maximum (i.e., in the early morning at the CBT minimum), under high homeostatic sleep pressure (i.e., after more than $16 \mathrm{~h}$ of wakefulness).

The impact of light on sleep and wakefulness, however, does not happen in a homogenous manner for all types of light exposure, but intimately depends on intensity, timing, duration, and wavelength. Thus, in the next sections, we describe how these aspects of light exposure can play a crucial role on wakefulness.

\section{Do light effects impact on sleep and wakefulness irrespective of timing?}

While applying light during sleep is difficult in humans, measuring light effects during wakefulness is easy, but its interpretation rather multifaceted, since factors such as the duration of prior wakefulness, endogenous circadian phase, and prior environmental light exposure all interact with each other.

Most studies on the effects of light in humans are conducted at night (Badia et al., 1991; Campbell and Dawson 1990; Foret et al., 1996; Lockley et al., 2006). Considering how light acts on the circadian and homeostatic systems (as described in the previous section), one does in fact expect that, even during the biological night in extended wakefulness (when sleep pressure is rising), light can dramatically enhance subjective alertness and reduce objective markers of sleepiness (e.g., slow eye movements). From a circadian perspective, there is a considerable body of evidence that suggests that, at night, the strong melatonin suppression caused by exposure to highintensity light may be one of the underlying mechanisms (Cajochen et al., 1998; Sack et al., 1992). Although not consistently shown, it has 
been hypothesized that melatonin attenuates $\mathrm{SCN}$-dependent mechanisms responsible for promoting and maintaining cortical and behavioral arousal at particular times in the circadian cycle (Cajochen et al., 1999a,b; Dijk and Czeisler 1995; Sack et al., 1997; Wright, 1997).

However, it is very likely that there are additional mechanisms that mediate the alerting effects of light. Light exposure at night on the nasal part of the retina does induce suppression of melatonin, without effects on alertness (Ruger et al., 2006). Also during the biological day, when melatonin is at minimal levels, light does impact on alertness. A study in which participants were exposed to either bright light (5000 lux) or dim light $<10$ lux (control condition) either between 12:00 and 16:00 $\mathrm{h}$ or between 00:00 and 04:00 $\mathrm{h}$ showed that bright light had a time-dependent effect on heart rate and CBT, such that brightlight exposure at night, but not in daytime, increased heart rate and increased CBT. However, the effects of bright light on the psychological variables was time independent, since both nighttime and daytime bright light reduced sleepiness and fatigue significantly and similarly (Fig. 1a and b; Ruger et al., 2006).

Further evidence in support of daytime effects of light effects on alertness was found in an "inlab" study, with daytime exposure to short (21 min) white light at $>7000$ lux; cortical activity was enhanced during an oddball task and subjective alertness improved in a dynamic manner (Vandewalle et al., 2006). This suggests that light may modulate activity of subcortical structures involved in alertness, thereby promoting cortical activity in networks involved in ongoing nonvisual cognitive processes.

It remains inconclusive and controversial whether the timing of light exposure does have an impact on human sleep. For instance, in an ultrashort sleep-wake schedule (Kubota et al., 2002), exposure to evening bright light (5000 lux) delayed the diurnal fluctuation of sleep propensity, which suggests that light may have the potential to phase-advance or -delay sleep phase in a similar manner as to the phase-response curve (PRC) derived from CBT-or melatonin-rhythm. Melatonin has been hypothesized to act as a mediator to convey the output of the circadian pacemaker to the sleep-wake system (Lavie, 1997). Nocturnal bouts of sleep propensity observed in an ultrashort sleep-wake schedule seem to occur in parallel with an increase in melatonin secretion near to habitual bedtime (Kubota et al., 2002). However, it is also likely that the magnitude of the phase change in melatonin secretion after bright-light exposure may not correlate with that in sleep propensity rhythm. An alternative hypothesis suggests that an indirect effect of melatonin on sleep-wake cycle mediated via temperature may be essential in considering coupling mechanisms between the circadian pacemaker and sleep, since melatonin acts strongly on the temperature rhythm (Van Someren, 2000).

However, timing per se is not the only factor one should bear in mind when considering how light impacts on alertness. Since the aforementioned studies used polychromatic light above 1000 lux, it may be that the intensity of the light exposure is, in fact, responsible for such alerting effects, which appear to be irrespective of timerelated dependency. This leads to the next question: What is the threshold of light intensity that can keep us awake?

\section{Dose-response effects of light: Is there a saturation point?}

Light is a powerful synchronizer which resets the endogenous circadian pacemaker to the 24-h day in an intensity-dependent manner. Although it is clearly recognized that bright light (1000 lux or more) is an effective synchronizer in humans, one might believe that the human circadian pacemaker is insensitive to lower levels of light illumination (i.e., <100 lux). However, the relationship between the resetting effect of light and its intensity follows a compressive nonlinear function, 
Comp. by: PG1361AAruldurai Stage: Proof ChapterID: 0001253860PBR978-0-444-53817-8 Date:4/

3/11 Time:20:33:13

File Path:I|pchns1002zIWOMATIProduction|PRODENV1000000000110000025244|0000000016

$10001253860.3 d$

Acronym:PBR Volume:19007
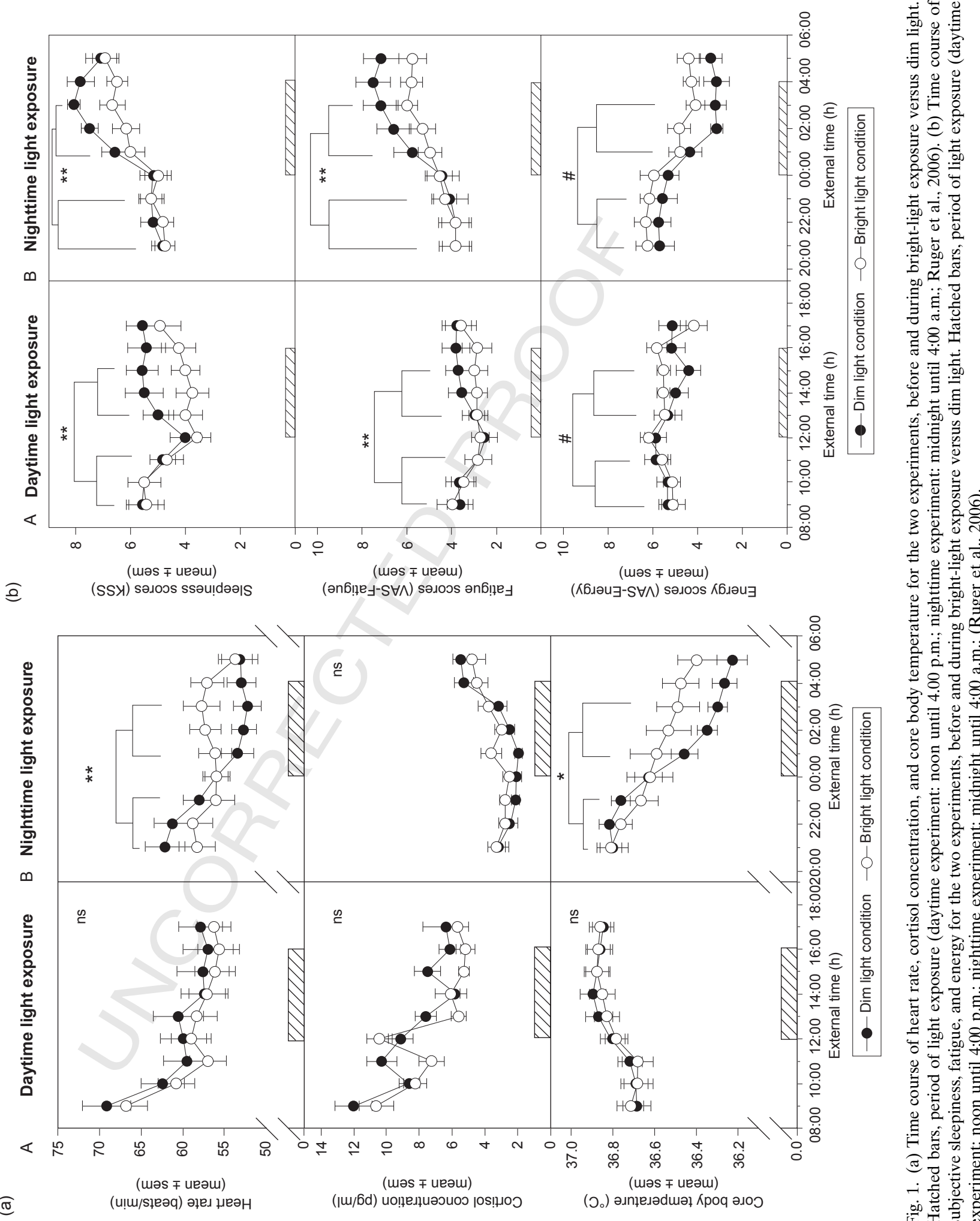
such that exposure to lower illuminances still elicits a strong effect (Boivin et al., 1996). Indeed, the dose-response function to a single episode of light prior to CBT nadir is such that $50 \%$ of the maximal resetting response to bright light at 9100 lux can be obtained with dim room light (100 lux; Cajochen et al., 2000; Zeitzer et al., 2000; Fig. 2). Further, humans have the capacity to keep stable entrainment to a $24-\mathrm{h}$ cycle even when ambient light levels are around 1.5 lux, which suggests that also low-lit environments can induce small shifts in the circadian system (Duffy and Wright 2005).

The phase-shifting dose-response function to light is fairly similar to the dose-response function for the alertness enhancing effects of light (Zeitzer et al., 2000). In other words, nighttime exposure to typical room light can exert an alerting effect in humans, as indexed by lower subjective ratings of sleepiness, less slow eye movements, and less theta and alpha waking

(a)

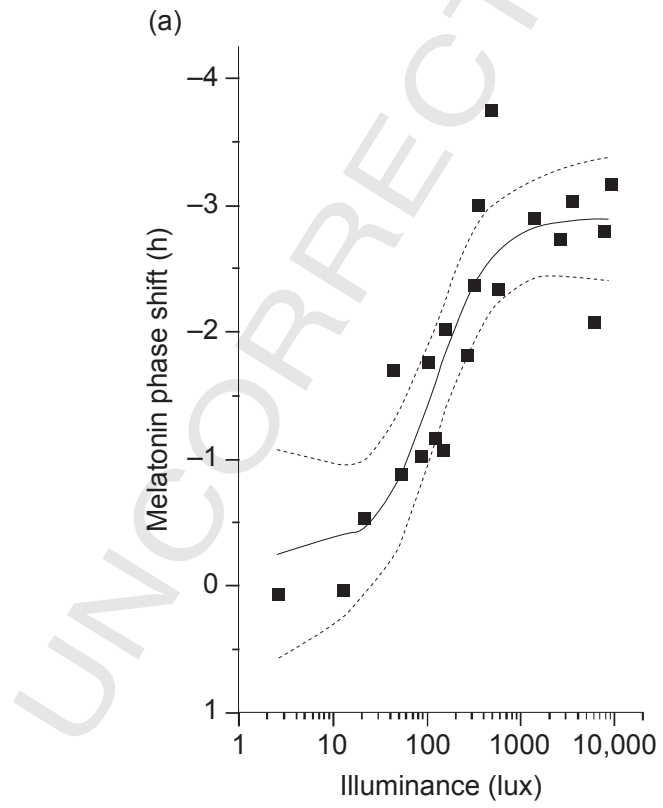

EEG activity. Taken together, this implies that the answer for the title of this section is yesthere is a saturation point for light impacts on alertness, and this high sensitivity may explain why, sometimes, a direct effect of light has not been observed, given that these light effects were compared to dim light sufficient to elicit near maximal effects (Dollins et al., 1993; Myers and Badia 1993).

If even low-light intensities can keep us awake, could it be that the duration of light exposure and/or our prior light history also matter and not only the intensity of light? In the next section, we will delineate some possible answers.

\section{Duration of light and prior light exposure}

A recent systematic evaluation of the duration dependence of the circadian resetting responses of a single dose of light in mice shows that light

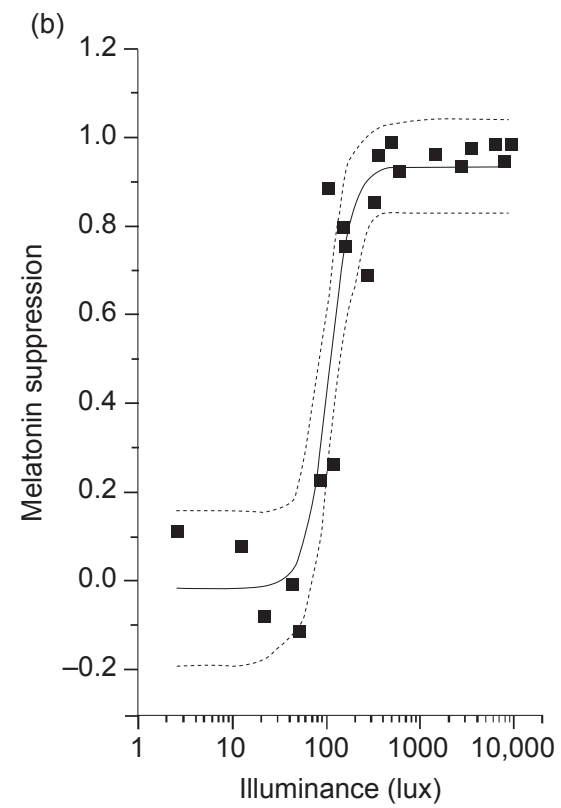

Fig. 2. Illuminance-response curve of the human circadian pacemaker. (a) illustrates the phase shift of melatonin rhythm, as assessed on the day following exposure to a $6.5 \mathrm{~h}$ experimental light stimulus, while (b) illustrates the magnitude of melatonin suppression during the light exposure (Zeitzer et al., 2000). 
pulse duration affects both amplitude and shape of the PRC (Comas et al., 2006). Using a model in which only phase shifts but no period or amplitude changes to light pulses were included, the authors concluded that phase-shifting effects are largest in the first hour of the light pulse, and reduce to a factor 0.22 during all hours after the first hour. Similar conclusions were drawn from an analysis of available data in humans (Beersma et al., 2009). Analyses of human PRC indicate that during exposure to $6.5 \mathrm{~h}$ of bright white light ( $\sim 10,000$ lux $)$, phase delays occurred when light was centered before the critical phase at CBT minimum, while phase advances occurred when light fell after the critical phase (Khalsa et al., 2003). Exposure to intermittent light also seems to be highly effective at resetting the human circadian system. The phase-resetting effects of $5 \mathrm{~h}$ of continuous bright white light $(\sim 10,000$ lux $)$ are comparable to a 5-intermittent exposure of six cycles of $15 \mathrm{~min}$ of bright light $(\sim 10,000 \mathrm{lux}$; Gronfier et al., 2004). Thus, a single sequence of intermittent bright-light pulses has a greater resetting efficacy on a per-minute basis than does continuous light exposure. This has been explained by a response saturation process, which is fundamental to proper functioning of the circadian pacemaker in a natural environment (Beersma et al., 2009). In a subsequent study, exposure to two 45-min pulses of bright light in the early subjective evening entrained the circadian system to a non-24-h day, again indicating that intermittent pulses are highly efficient at resetting human circadian rhythms (Gronfier et al., 2007).

Response saturation plays a role in phase resetting to long light pulses, but also adaptation to light clearly affects phase-shifting responses to light. Prior exposure to high levels of light during periods varying from 3 days to 1 week attenuated suppression of the melatonin concentration at night in response to 200-500 lux of light, as compared to prior exposure to 3 days to 1 week of low levels or dim light (Smith et al., 2004). However, if preexposure to room light can desensitize circadian phase resetting or attenuates the alerting effects of light remains an open question.

\section{Short-wavelength effects: Conventional visual photoreception is not the key mediator}

The relationship between the wavelength of light and its alerting response seems to indicate a predominance of short-wavelength light (470 nm and lower) in comparison to other wavelengths (Lockley et al., 2006; Münch et al., 2006; Revell et al., 2006). Exposure to 460-nm (blue) monochromatic light for $6.5 \mathrm{~h}$ during the biological night (maximum levels of melatonin secretion) can substantially decrease both subjective sleepiness, improve cognitive performance in tasks of sustained attention (i.e., psychomotor vigilance task), and decrease waking EEG power density in the delta-theta frequency range (Fig. 3), when compared to light of equal photon density of 555-nm (green) monochromatic light (Lockley et al., 2006). The magnitude of greater responses following exposure to an equal number of photons of 460-nm light, as compared with 555-nm light, strongly suggests that the photoreceptors mediating these acute effects of light are blue shifted with respect to the visual photopic system. Similarly, a 2-h evening exposure to monochromatic light of two different wavelengths (460 and $550 \mathrm{~nm}$ ) at very low intensities resulted in more alertness during exposure at $460 \mathrm{~nm}$, which further suggests a blue-shift response to light (Revell et al., 2006). However, these responses do not confine only to effects on wakefulness, but have been observed on a wide array of physiological variables, such as melatonin suppression (Lewy et al., 1980; Zeitzer et al., 2000), circadian phase shifting (Cajochen et al., 1992), nocturnal decline in EEG SWA (Münch et al., 2006; Fig. 4), and circadian gene expression (PER2) in oral mucosa (Cajochen et al., 2006). Nevertheless, novel evidence supports that cone photoreceptors may also contribute to NIF responses at the 

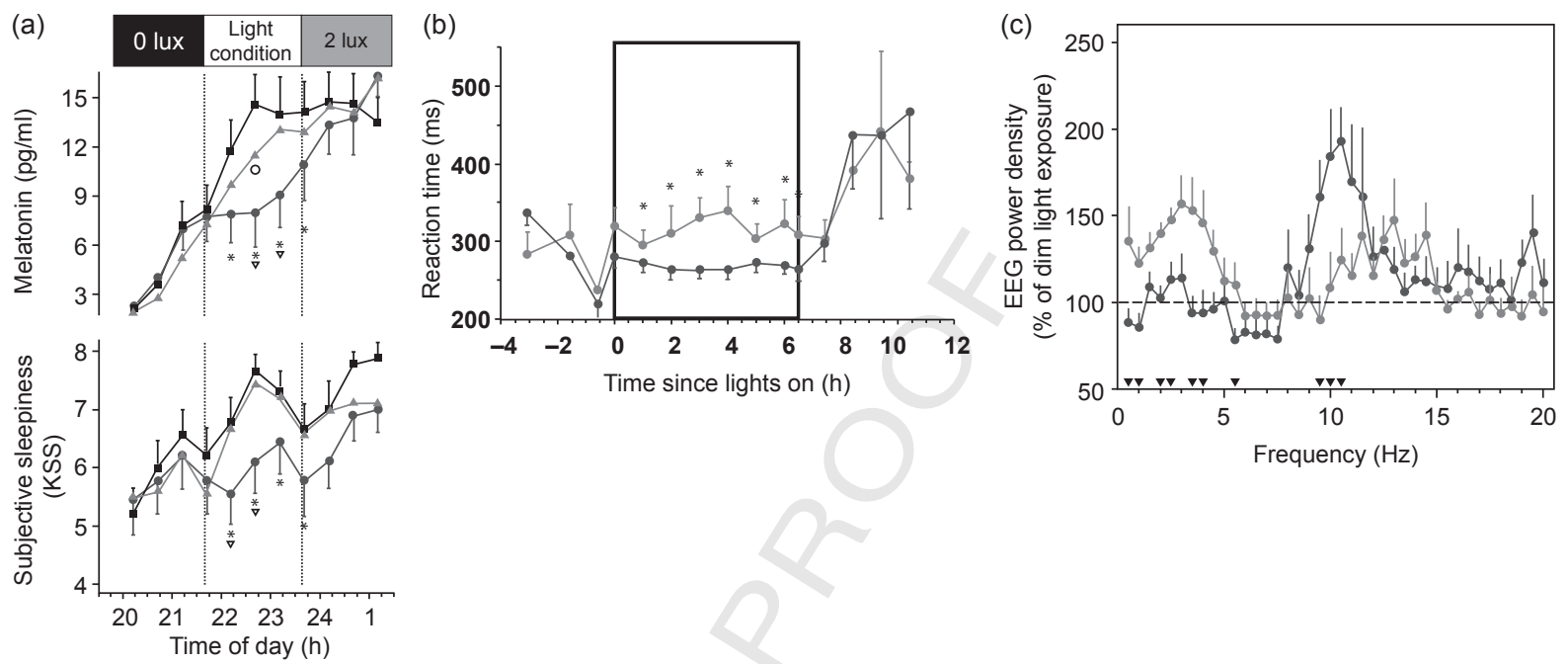

Fig. 3. Effect of light exposures on melatonin secretion, subjective sleepiness, performance, and the electroencephalogram in young healthy volunteers. (a) Effects of a 2-h darkness period (black line) and of 2-h monochromatic light exposures at $460 \mathrm{~nm}$ (blue line) and $550 \mathrm{~nm}$ (green line) in the evening on salivary melatonin levels and subjective sleepiness (Cajochen et al., 2006); (b) continuous $6.5 \mathrm{~h}$ nighttime exposure to blue monochromatic light (significantly improved auditory reaction times to a simple vigilance task when compared to green monochromatic light; Lockley et al., 2006); (c) EEG power density during continuous $6.5 \mathrm{~h}$ nighttime exposure to monochromatic blue light and monochromatic green light (Lockley et al., 2006). (For interpretation of the references to color in this figure legend, the reader is referred to the Web version of this chapter.)

beginning of a light exposure and at low irradiances, while melanopsin can be the primary circadian photopigment in response to long-duration light exposure and at high irradiances (Gooley et al., 2010).

The neurophysiology underpinnings that account for light-induced responses are still not fully comprehended. It is known that intrinsically photosensitive retinal ganglion cells (ipRGCs) project to a range of targets, including the $\mathrm{SCN}$, subparaventricular zone, and the pretectal area that are implicated in mediating NIF responses (Hattar et al., 2002). Further, these cells also project directly to the ventrolateral preoptic area, a hypothalamic nucleus lateral to the optic chiasm and rostral to the SCN that also receive secondary afferents from the SCN, subparaventricular zone, and dorsomedial hypothalamus (Hattar et al., 2002). Ventrolateral preoptic area innervates all of the major nuclei of the ascending monoaminergic and, in particular, the histaminergic pathways, which are thought to play a key role in wakefulness and EEG arousal (Aston-Jones et al., 1999; Lin et al., 1996). Direct photic input to this nucleus may therefore alter ventrolateral preoptic area activity. The locus coeruleus (LC) located in dorsal tegmentum of the pons is also involved in the regulation of the sleep-wake cycle (Saper et al., 2005) regulating the amplitude of the sleep-wake circadian rhythm set by the SCN by increasing wakefulness during the active period (Gonzalez and Aston-Jones 2006).

Alternatively, the acute alerting effects of light may happen through acute melatonin suppression, given that increased melatonin suppression can be associated with greater arousal and/or attenuation of the endogenous circadian drive for alertness (Lockley et al., 2006). In other words, if short-wavelength light can reduce circulating melatonin levels and/or high-frequency 


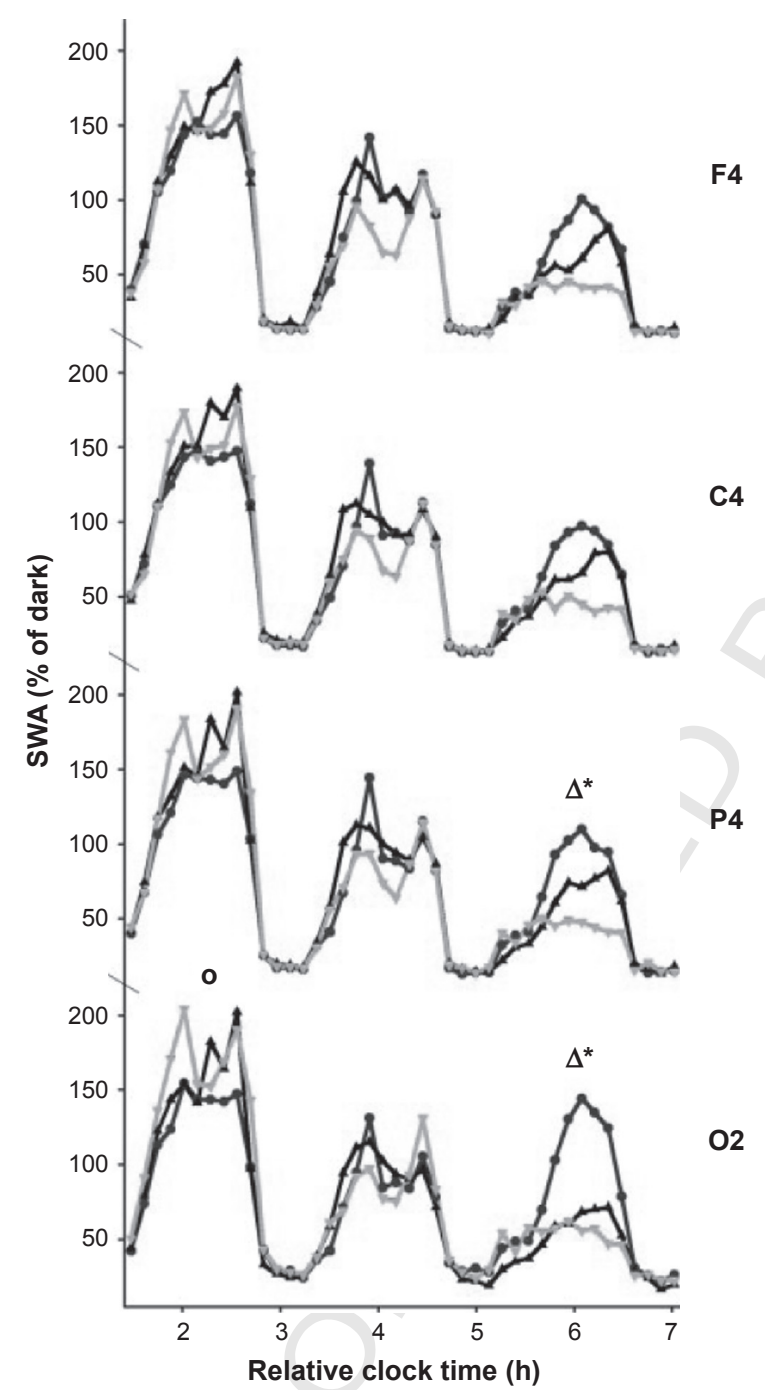

Fig. 4. Dynamics of SWA per NREM-REM sleep cycles 1-3 after sleep onset for EEG derivations F4, C4, P4, and O2. Values are expressed as percentages of the dark condition and are plotted against relative clock time $(n=8)$ for blue light $(460 \mathrm{~nm}$, blue circle), green light $(550 \mathrm{~nm}$, green triangle, down), and dark condition (0 lux, black triangle). $* P<0.05 ;{ }^{\circ} P<0.1$, green light versus blue light; ${ }^{\Delta} P<0.05$, blue light versus dark condition (Münch et al., 2006). (For interpretation of the references to color in this figure legend, the reader is referred to the Web version of this chapter.) alpha activity $(9.25-12.0 \mathrm{~Hz}$; a marker of the endogenous circadian drive for alertness), it can enhance subjective and objective arousal states. This assumption seems to hold true, given a number of studies which show a reduction of either or both under blue-light exposure (Lockley et al., 2006; Münch et al., 2006). Nevertheless, it is also quite likely that, while melatonin could have a direct role in mediating alertness during the biological night, there might be alternative pathways. For instance, in contrast to Lockley et al. (2006), Phipps-Nelson et al. (2009) did not find blue-light exposure effects on EEG alpha activity, subjective sleepiness, or salivary melatonin, while they observed differential response on the delta/theta activity. These discrepancies may be explained by the fact that in this study, the intensity of blue light was of $2.1 \mu \mathrm{W} / \mathrm{cm}^{2}$, while the other study used a much higher intensity $\left(12.1 \mu \mathrm{W} / \mathrm{cm}^{2}\right)$. Exposure to $460 \mathrm{~nm}$ light at $3.1 \mu \mathrm{W} / \mathrm{cm}^{2}$ resulted in significant suppression of plasma melatonin (Brainard et al., 2001), whereas exposure to the same wavelength at $\leq 2.3 \mu \mathrm{W} / \mathrm{cm}^{2}$ did not result in significant suppression. Thus, one might speculate that if the irradiance of the bluelight $(460 \mathrm{~nm})$ stimulus is below the threshold of irradiance required for melatonin suppression, other systems may, in turn, undergo light effects, such as the homeostatic rather than circadian mechanisms (Lockley and Gooley 2006).

In the next section, we now turn to what are the cerebral correlates of light that can play a pivotal role on cognitive performance.

\section{Cerebral correlates of light impacts' on cognitive performance}

The effect of light on cognitive performance impinges on subcortical and cortical regions in a differential way. Light modulations on cortical activity during auditory cognitive tasks affect alertness-related subcortical structures, such 
as the brainstem (LC-compatible region; Vandewalle et al., 2007a,b); the hypothalamus, in a location encompassing the SCN (Perrin et al., 2004), and dorsal and posterior parts of thalamus (Vandewalle et al., 2006, 2007), in long-term memory and emotion-related areas, such as the hippocampus (Vandewalle et al., 2006) and amygdala (Vandewalle et al., 2007a,b). As can be observed, these responses point to a wide-range of subcortical and cortical regions that are differentially activated by the nonvisual effects of light, during specific cognitive tasks. Further, fMRI assessed brain responses undergo a wavelength dependency for higher executive task (two-back task), such that blue light enhances modulations in the brainstem (in an LCcompatible location), in the thalamus and insula, in relation to green $(550 \mathrm{~nm})$ and violet exposures $(430 \mathrm{~nm})$. In this case, the effects of blue light occur $1 \mathrm{~min}$ after the start of the exposure (Vandewalle et al., 2007a,b) and lasted for $\sim 20 \mathrm{~min}$ (Vandewalle et al., 2007a,b). Nonetheless, the degree, temporal dynamics, and regional brain distribution of nonvisual effects of light crucially relies on light properties, such as dose, duration, and intensity. Contrary to subcortical regions, which are activated faster and show short-lasting responses to light, long-lasting and widespread task-related responses occur only when light exposure has a longer duration and at a higher intensity (Perrin et al., 2004). For instance, exposure to $20 \mathrm{~min}$ of bright white light has been shown to induce thalamic and cortical modulations that steadily declined after light exposure despite the lasting effects (responses were observed after several minutes of the end of the light exposure; Vandewalle et al., 2006). However, when light exposure duration was less than a minute, the majority of effects were elicited for subcortical structures, such as the dorso-posterior thalamus and the brainstem (LC-compatible area; Vandewalle et al., 2007a,b).

The overarching significance of LC-related areas in this case is due to its projections to numerous cortical sites, which favors its role as a mediator for light changes in alertness and cognitive performance (Gonzalez and Aston-Jones 2006). The thalamus, in particular, its dorsal and posterior nuclei (i.e., pulvinar), is a key structure involved in the interaction between alertness and cognition (Portas et al., 1998), and lightrelated changes in its activity can be directly implicated in enhanced alertness during light exposure. Bearing in mind that the thalamus plays a critical role in the relay of information to the cortex, effects of light on the thalamus may result in widespread cortical effects.

Interestingly, the effects of monochromatic blue light on the hippocampus and amygdala followed a dissimilar pattern (Vandewalle et al., 2007a,b), as responses in these limbic structures happened almost immediately after light onset. These swift limbic light-induced responses might have occurred due to the anatomical connectivity of these structures. The amygdala receives direct inputs from ipRGCs and indirect retinal inputs through the superior colliculus and thalamus. In turn, the amygdala has direct projections to the hippocampus, which receives activating inputs from the brainstem (Vandewalle et al., 2009). The functional relevance of these limbic responses remains uncertain, but raises the question if blue light might support an early affective and mnemonic arousal, which enables a prompt behavioral adaptation to the environment. Similarly, the light-induced modulation of amygdala activity may correspond to one of the underlying reasons for the therapeutical property of light in mood disorders. Light therapy is the treatment of choice of seasonal affective disorder (SAD), and the relative contribution of blue light to overall natural light exposure is smaller during the winter than during the summer (Thorne et al., 2009). However, it is still uncertain as to whether the longterm effects of repeated exposures on mood as used in light therapy are related to the acute modulation of brain activity to tasks that do not involve emotion. 


\section{Summary}

Light exerts dominant nonvisual effects on numerous physiological variables, such as the human sleep-wake cycle and cognitive performance, primarily through properties such as dose, duration, timing, and wavelength. The use of reliable and sensitive methods such as fMRI to evaluate light-induced nonvisual brain responses have increased our understanding on how light can optimize brain function during specific cognitive tasks. These stimulating discoveries will certainly help to unravel how retinal and suprachiasmatic networks modulate the complex interplay of circadian rhythms, sleep-wake homeostasis, and cognition.

\section{References}

Albrecht, U., Zheng, B., et al. (2001). MPer1 and mPer2 are essential for normal resetting of the circadian clock. Journal of Biological Rhythms, 16(2), 100-104.

Aston-Jones, G., Rajkowski, J., et al. (1999). Role of locus coeruleus in attention and behavioral flexibility. Biological Psychiatry, 46, 1309-1320.

Badia, P., Myers, B., et al. (1991). Bright light effects on body temperature, alertness, EEG and behavior. Physiology \& Behavior, 50, 583-588.

Beersma, D. G. M., Comas, M., et al. (2009). The progression of circadian phase during light exposure in animals and humans. Journal of Biological Rhythms, 24(2), 153-160.

Berson, D. M. (2003). Strange vision: Ganglion cells as circadian photoreceptors. Trends in Neurosciences, 26, 314-320.

Boivin, D. B., Duffy, J. F., et al. (1996). Dose-response relationships for resetting of human circadian clock by light. Nature, 379, 540-542.

Borbély, A. A. (1982). A two process model of sleep regulation. Human Neurobiology, 1, 195-204.

Brainard, G., Hanifin, J. P., et al. (2001). Human melatonin regulation is not mediated by the three cone photopic visual system. The Journal of Clinical Endocrinology and Metabolism, 86, 433-436.

Bromundt, V., Köster, M., et al. (2010). Sleep-wake cycles and cognitive functioning in schizophrenia. The British Journal of Psychiatry, 197, .

Burgess, H. J., Sharkey, K. M., et al. (2002). Bright light, dark and melatonin can promote circadian adaptation in night shift workers. Sleep Medicine Reviews, 6(5), 407-420.
Buysse, D. J., Barzansky, B., et al. (2003). Sleep, fatigue, and medical training: Setting an agenda for optimal learning and patient care. Sleep, 26, 218-225.

Cajochen, C., Dijk, D. J., et al. (1992). Dynamics of EEG slow-wave activity and core body temperature in human sleep after exposure to bright light. Sleep, 15, 337-343.

Cajochen, C., Jud, C., et al. (2006). Evening exposure to blue light stimulates the expression of the clock gene PER2 in humans. The European Journal of Neuroscience, 23, 1082-1086.

Cajochen, C., Khalsa, S. B. S., et al. (1999a). EEG and ocular correlates of circadian melatonin phase and human performance decrements during sleep loss. American Journal of Physiology. Regulatory, Integrative and Comparative Physiology, 277, R640-R649.

Cajochen, C., Kräuchi, K., et al. (1998). Evening administration of melatonin and bright light: Interactions on the EEG during sleep and wakefulness. Journal of Sleep Research, 7, 145-157.

Cajochen, C., Münch, M., et al. (2005). High sensitivity of human melatonin, alertness, thermoregulation and heart rate to short wavelength light. The Journal of Clinical Endocrinology and Metabolism, 90, 1311-1316.

Cajochen, C., Zeitzer, J. M., et al. (1999b). Dose-response relationship for light intensity and alertness and its ocular and EEG correlates. Sleep Research Online, 2, 517.

Cajochen, C., Zeitzer, J. M., et al. (2000). Dose- response relationship for light intensity and ocular and electroencephalographic correlates of human-alertness. Behavioural Brain Research, 115, 75-83.

Campbell, S. S., \& Dawson, D. (1990). Enhancement of nighttime alertness and performance with bright ambient light. Physiology \& Behavior, 48, 317-320.

Carrier, J., \& Dumont, M. (1995). Sleep propensity and sleep architecture after bright light exposure at three different times of day. Journal of Sleep Research, 4, 202-211.

Comas, M., Beersma, D. G. M., et al. (2006). Phase and period responses of the circadian system of mice (Mus musculus) to light stimuli of different duration. Journal of Biological Rhythms, 21(5), 362-372.

Czeisler, C. A., Dijk, D. J., et al. (1994). Entrained phase of the circadian pacemaker serves to stabilize alertness and performance throughout the habitual waking day. In R. D. Ogilvie \& J. R. Harsh (Eds.), Sleep onset: Normal and abnormal processes (pp. 89-110). Washington, DC: Au9 American Psychological Association.

Czeisler, C. A., \& Gooley, J. (2007). Sleep and circadian rhythms in humans. Cold Spring Harbor Symposia on Quantitative Biology, 72, 579-597.

Daan, S., Beersma, D. G. M., et al. (1984). Timing of human sleep: Recovery process gated by a circadian pacemaker. The American Journal of Physiology, 246, R161-R178. 
Daurat, A., Foret, J., et al. (2000). Bright light during nighttime: Effects on the circadian regulation of alertness and performance. Biological Signals and Receptors, 9, 309-318.

Dijk, D. J., Beersma, D. G. M., et al. (1989). Bright morning light advances the human circadian system without affecting NREM sleep homeostasis. American Journal of Physiology. Regulatory, Integrative and Comparative Physiology, 256, R106-R111.

Dijk, D. J., \& Czeisler, C. A. (1994). Paradoxical timing of the circadian rhythm of sleep propensity serves to consolidate sleep and wakefulness in humans. Neuroscience Letters, 166, 63-68.

Dijk, D. J., \& Czeisler, C. A. (1995). Contribution of the circadian pacemaker and the sleep homeostat to sleep propensity, sleep structure, electroencephalographic slow waves, and sleep spindle activity in humans. The Journal of Neuroscience, 15, 3526-3538.

Dijk, D. J., Shanahan, T. L., et al. (1997). Variation of electroencephalographic activity during non-rapid eye movement and rapid eye movement sleep with phase of circadian melatonin rhythm in humans. The Journal of Physiology, $505,851-858$

Dollins, A. B., Lynch, H. J., et al. (1993). Effects of illumination on human nocturnal serum melatonin levels and performance. Physiology \& Behavior, 53, 153-160.

Duffy, J. F., \& Dijk, D. J. (2002). Getting through to circadian oscillators: Why use constant routines? Journal of Biological Rhythms, 17(1), 4-13.

Duffy, J. E., \& Wright, K. P. (2005). Entrainment of the human circadian system by light. Journal of Biological Rhythms, 20(4), 326-338.

Edelstein, K., de la Iglesia, H. O., et al. (2003). Behavioral arousal blocks light-induced phase advances in locomotor rhythmicity but not light-induced Per1 and Fos expression in the hamster suprachiasmatic nucleus. Neuroscience, 118 (1), 253-261.

Foret, J., Daurat, A., et al. (1996). The effect on body temperature and melatonin of a 39-h constant routine with two different light levels at nighttime. Chronobiology International, $13,35-45$.

Giménez, M. C., Hessels, M., et al. (2010). Effects of artificial dawn on subjective ratings of sleep inertia and dim light melatonin onset. Chronobiology International, 27(6), 1219-1241.

Gonzalez, M. M., \& Aston-Jones, G. (2006). Circadian regulation of arousal: Role of the noradrenergic locus coeruleus system and light exposure. Sleep, 29, 1327-1336.

Gooley, J. J., Rajaratnam, S. M. W., et al. (2010). Spectral responses of the human circadian system depend on the irradiance and duration of exposure to light. Science Translational Medicine, 2(31), 31ra33.

Gordijn, M. C. M., Beersma, D. G. M., et al. (1999). Effects of light exposure and sleep displacement on dim light melatonin onset. Journal of Sleep Research, 8(3), 163-174.
Gronfier, C., Wright, K. P. Jr., et al. (2004). Efficacy of a single sequence of intermittent bright light pulses for delaying circadian phase in humans. American Journal of Physiology. Endocrinology and Metabolism, 287(1), E174-E181.

Gronfier, C., Wright, K. P. Jr., et al. (2007). Entrainment of the human circadian pacemaker to longer-than-24-h days. Proceedings of the National Academy of Sciences of the United States of America, 104, 9081-9086.

Guler, A. D., Ecker, J. L., et al. (2008). Melanopsin cells are the principal conduits for rod-cone input to non-imageforming vision. Nature, 453(7191), 102-105.

Hatori, M., \& Panda, S. (2010). The emerging roles of melanopsin in behavioral adaptation to light. Trends in Molecular Medicine, 16(10), 435-446.

Hattar, S., Liao, H. W., et al. (2002). Melanopsin-containing retinal ganglion cells: Architecture, projections, an intrinsic photosensitivity. Science, 295, 1065-1071.

Khalsa, S. B. S., Jewett, M. E., et al. (2003). A phase response curve to single bright light pulses in human subjects. The Journal of Physiology, 549(3), 945-952.

Kleitman, N. (1987). Sleep and wakefulness. Chicago and London: The University of Chicago Press.

Koorengevel, K. M., Beersma, D. G. M., et al. (2003). Mood regulation in seasonal affective disorder patients and healthy controls studied in forced desynchrony. Psychiatry Research, 117, 57-74.

Kubota, T., Uchiyama, M., et al. (2002). Effects of nocturnal bright light on saliva melatonin, core body temperature and sleep propensity rhythms in human subjects. Neuroscience Research, 42, 115-122.

Lavie, P. (1997). Melatonin: Role in gating nocturnal rise in sleep propensity. Journal of Biological Rhythms, 12, 657-665.

Lavoie, S., Paquet, J., et al. (2003). Vigilance levels during and after bright light exposure in the first half of the night. Chronobiology International, 20, 1019-1038.

Lewy, A. J., Wehr, T. A., et al. (1980). Light suppresses melatonin secretion in humans. Science, 210, 1267-1269.

Lin, J. S., Hou, Y., et al. (1996). Histaminergic descending inputs to the mesopontine tegmentum and their role in the control of cortical activation and wakefulness in the cat. The Journal of Neuroscience, 16, 1523-1537.

Lockley, S. W., Evans, E. E., et al. (2006). Short-wavelength sensitivity for the direct effects of light on alertness, vigilance, and the waking electroencephalogram in humans. Sleep, 29, 161-168.

Lockley, S. W., \& Gooley, J. J. (2006). Circadian photoreception: Spotlight on the brain. Current Biology, 16, R795-R797.

Lupi, D., Oster, H., et al. (2008). The acute light-induction of sleep is mediated by OPN4-based photoreception. Nature Neuroscience, 11(9), 1068-1073.

Moseley, M., Bayliss, S., et al. (1988). Light transmission through the human eyelid: In vivo measurement. Ophthalmic \& Physiological Optics, 8(2), 229-230. 
Münch, M., Kobialka, S., et al. (2006). Wavelength-dependent effects of evening light exposure on sleep architecture and sleep EEG power density in men. American Journal of Physiology: Regulatory, Integrative and Comparative Physiology, 290, R1421-R1428.

Muñoz, M., Peirson, S. N., et al. (2005). Long - term constant light induces constitutive elevated expression of mPER2 protein in the murine SCN. A molecular basis for Aschoffs rule? Journal of Biological Rhythms, 20, 3-14.

Myers, B. L., \& Badia, P. (1993). Immediate effects of different light intensities on body temperature and alertness. Physiology \& Behavior, 54, 199-202.

Oosterman, J. M., Van Someren, E. J. W., et al. (2009). Fragmentation of the rest-activity rhythm correlates with agerelated cognitive deficits. Journal of Sleep Research, 18(1), 129-135.

Oster, H., Maronde, E., et al. (2002). The circadian clock as a molecular calendar. Chronobiology International, 19(3), 507-516.

Perrin, F., Peigneux, P., et al. (2004). Nonvisual responses to light exposure in the human brain during the circadian night. Current Biology, 14, 1842-1846.

Phipps-Nelson, J., Redman, J. R., et al. (2009). Blue light exposure reduces objective measures of sleepiness during prolonged nighttime performance testing. Chronobiology International, 26(5), 891-912.

Portas, C. M., Rees, G., et al. (1998). A specific role for the thalamus in mediating the interaction of attention and arousal in humans. The Journal of Neuroscience, 18(21), 8979-8989.

Revell, V. L., Arendt, J., et al. (2006). Alerting effects of light are sensitive to very short wavelengths. Neuroscience Letters, 399(1-2), 96-100.

Riemersma-van der Lek, R. F., Swaab, D. F., et al. (2008). Effect of bright light and melatonin on cognitive and noncognitive function in elderly residents of group care facilities: A randomized controlled trial. The Journal of the American Medical Association, 299(22), 2642-2655.

Ruger, M., Gordijn, M. C. M., et al. (2006). Time-of-daydependent effects of bright light exposure on human psychophysiology: Comparison of daytime and nighttime exposure. American Journal of Physiology: Regulatory, Integrative and Comparative Physiology, 290(5), R1413-R1420.

Rusak, B., Robertson, H., et al. (1990). Light pulses that shift rhythms induce gene expression in the suprachiasmatic nucleus. Science, 248(4960), 1237-1240.

Sack, R. L., Blood, M. L., et al. (1992). Oral melatonin reverses the alerting effects of nocturnal bright light exposure in humans. Sleep Research, 21, 49.

Sack, R. L., Hughes, R. J., et al. (1997). Sleep-promoting effects of melatonin: At what dose, in whom, under what conditions, and by what mechanisms? Sleep, 20, 908-915.
Santhi, N., Aeschbach, D., et al. (2008). The impact of sleep timing and bright light exposure on attentional impairment during night work. Journal of Biological Rhythms, 23(4), 341-352.

Saper, C. B., Scammell, T. E., et al. (2005). Hypothalamic regulation of sleep and circadian rhythms. Nature, 437(7063), 1257-1263.

Smith, K. A., Schoen, M. W., et al. (2004). Adaptation of human pineal melatonin suppression by recent photic history. The Journal of Clinical Endocrinology and Metabolism, 89(7), 3610-3614.

Thorne, H. C., Jones, K. H., et al. (2009). Daily and seasonal variation in the spectral composition of light exposure in humans. Chronobiology International, 26(5), 854-866.

Tsai, J. W., Hannibal, J., et al. (2009). Melanopsin as a sleep modulator: Circadian gating of the direct effects of light on sleep and altered sleep homeostasis in Opn4(-/-) mice. PLoS Biology, 7(6), e1000125.

Van Someren, E. (2000). More than a marker: Interaction between the circadian regulation of temperature and sleep, age-related changes, and treatment possibilities. Chronobiology International, 17(3), 313-354.

Vandewalle, G., Balteau, E., et al. (2006). Daytime light exposure dynamically enhances brain responses. Current Biology, 16(16), 1616-1621.

Vandewalle, G., Gais, S., et al. (2007a). Wavelength-dependent modulation of brain responses to a working memory task by daytime light exposure. Cerebral Cortex, Epub ahead of print.

Vandewalle, G., Maquet, P., et al. (2009). Light as a modulator of cognitive brain function. Trends in Cognitive Sciences, 13 (10), 429-438.

Vandewalle, G., Schmidt, C., et al. (2007b). Brain responses to violet, blue, and green monochromatic light exposures in humans: Prominent role of blue light and the brainstem. PLoS One, 2(11), e1247.

Werken, M., Giménez, M., et al. (2010). Effects of artificial dawn on sleep inertia, skin temperature, and the awakening cortisol response. Journal of Sleep Research, 19(3), 425-435.

Wright, K. P., Jr. (1997). Combination of bright light and caffeine as a countermeasure for impaired alertness and performance during extended sleep deprivation. Journal of Sleep Research, 6, 26-35.

Wright, K. P., Jr., Hull, J. T., et al. (2006). Sleep and wakefulness out of phase with internal biological time impairs learning in humans. Journal of Cognitive Neuroscience, 18, 508-521.

Zeitzer, J. M., Dijk, D. J., et al. (2000). Sensitivity of the human circadian pacemaker to nocturnal light: Melatonin phase resetting and suppression. The Journal of Physiology, $526,695-702$. 


\section{Query Form}

Book: Progress in Brain Research, 190

Chapter No: 07

\section{AU: Author Query; $\quad$ ED: Editor Query; $\quad$ TS: Query raised by Typesetter;}

\begin{tabular}{|c|c|c|}
\hline Query Refs. & Queries & Author's Response \\
\hline Au1 & $\begin{array}{l}\text { Note that the symbols "** }{ }^{* *}, \text { ns" are mentioned in the artwork of } \\
\text { Fig. } 1 \text { but its significance is missing in caption of Fig. } 1 \text {. Please } \\
\text { check. }\end{array}$ & \\
\hline Au2 & $\begin{array}{l}\text { Please provide telecommunication details and E-mail address for the } \\
\text { corresponding author "Marijke C. M. Gordijn". }\end{array}$ & \\
\hline Au3 & $\begin{array}{l}\text { As per in-house style "PER" have been italicized when referred to as } \\
\text { a gene. Please check. }\end{array}$ & \\
\hline Au4 & $\begin{array}{l}\text { Please check the introduction of slash in "desynchronized/ } \\
\text { synchronized subjects." }\end{array}$ & \\
\hline Au5 & $\begin{array}{l}\text { "Cajochen et al., 1999" has been changed to "Cajochen et al., } \\
\text { 1999a,b" as per reference list. Please check. }\end{array}$ & \\
\hline Au6 & Please check the edits made in the sentence "A study in which..." & \\
\hline Au7 & $\begin{array}{l}\text { As per style a maximum of up to six authors plus et al. should be } \\
\text { listed in the author group. Please list up to six authors for all the et al. } \\
\text { references. }\end{array}$ & \\
\hline Au8 & Please provide page range for this reference. & \\
\hline Au9 & $\begin{array}{l}\text { Please check whether publisher name is appropriate for this } \\
\text { reference. }\end{array}$ & \\
\hline Au10 & $\begin{array}{l}\text { Please check whether the inserted volume number and page range } \\
\text { for this reference is appropriate. }\end{array}$ & \\
\hline Au11 & Please provide volume number and page range for this reference. & \\
\hline
\end{tabular}

